\title{
Does gallbladder polyp size as measured using radiographic modalities predict pathological size in all types of polyps?
}

This article was published in the following Dove Press journal:

Reports in Medical Imaging

Jureerat Thammaroj'

Piti Ungarreevittaya ${ }^{2}$

Kriangsak Jenwitheesuk ${ }^{3,4}$

'Department of Radiology, Faculty of Medicine, Khon Kaen University, Khon Kaen, Thailand; ${ }^{2}$ Department of Pathology, Faculty of Medicine, Khon Kaen University, Khon Kaen, Thailand; ${ }^{3}$ Department of Surgery, Faculty of Medicine, Khon Kaen University, Khon Kaen, Thailand; ${ }^{4}$ Sleep Apnea Research Group, Khon Kaen University, Khon Kaen, Thailand
Correspondence: Kriangsak Jenwitheesuk Department of Surgery, Faculty of Medicine, Khon Kaen University, Khon Kaen 40002, Thailand

Tel +6643363133

Email surgerykkh@gmail.com
Background: Gallbladder polyps can be classified into two main types: cholesterol and adenoma. As polyp size is reported to be a factor suggestive for malignant polyps, this study aimed to evaluate whether radiographic size or any clinical factors are predictive of the pathological size of polyps.

Methods: This was a cross-sectional analytical study. The inclusion criteria were that patients had undergone laparoscopic cholecystectomy, had been diagnosed with gallbladder polyps using any radiographic modality, and had available pathological results. A clinical predictive model for pathological polyp size was calculated using multivariate linear regression analysis. Results: During the study period, there were 85 patients who met the study criteria. The average polyp size according to radiographic modalities was slightly larger than average (7.2 vs $5.6 \mathrm{~mm}$ ). The majority of polyps were cholesterol polyps (66; 77.6\%). The sizes of the polyps according to radiographic imaging were significantly associated with the pathological sizes of the polyps. Age was another significant factor related to the size of polyps identified using pathological methods in the adenoma group, with a coefficient of $0.264(P=0.021)$.

Conclusions: Gallbladder polyp size as determined using radiographic modalities can predict the pathological size in both cholesterol and adenoma polyps. Some adjustments are needed, particularly in patients with adenoma polyps.

Keywords: ultrasound, cholesterol polyp, adenoma, computed tomography, magnetic resonance cholangiopancreatography

\section{Introduction}

Gallbladder polyps are polypoid lesions in the gallbladder, and have a prevalence of approximately $5-7 \%$ in the adult population. ${ }^{1}$ The main clinical issue in cases of gallbladder polyps is differentiating among the types of polyps. ${ }^{2,3}$ The two major types are cholesterol and adenoma polyps. Polyps of the latter type are considered to be pre-malignant lesions and may need surgical intervention. Several studies have indicated that polyp size over $10 \mathrm{~mm}$ or in patients over 50 years of age may indicate a higher risk of malignancy., ${ }^{4,5}$

Ultrasonography is a radiographic modality frequently used to detect gallbladder polyps. ${ }^{6}$ However, the limitations of abdominal ultrasound include its low accuracy and inability to identify malignant polyps. A study from Turkey found that polyps were undetectable by ultrasound in 45 out of 82 patients $(55 \%) .{ }^{7}$ Another study also found that the positive predictive value of ultrasound for true polyp detection was low, at $0.18 .{ }^{8}$ In addition, high-resolution ultrasound may be able to detect malignant polyps using size, lobular surface, or vascular core. ${ }^{9}$ However, abdominal ultrasound is still a commonly 
used and widely available radiographic modality, particularly in resource-limited settings. As polyp size is reported to be a factor suggestive of malignant polyps, this study aimed to evaluate whether radiographic size or any clinical factors are predictive of the pathological size of polyps.

\section{Materials and methods}

This was a cross-sectional analytical study conducted in the Department of Surgery at the Khon Kaen University Faculty of Medicine in Thailand. The study period was between January and December 2017. The inclusion criteria were that patients had undergone laparoscopic cholecystectomy, had been diagnosed with gallbladder polyps using any radiographic modality, and had available pathological results. The charts of all eligible patients were retrospectively reviewed. Clinical data were collected, including baseline characteristics, symptoms of gallbladder polyps, risk factors for gallbladder polyps, laboratory results, and pathological results.

Patients were categorized into three groups: all patients, cholesterol polyps, and adenoma polyps. The cholesterol polyp group included patients with cholesterol polyps and/ or benign/chronic inflammation according to pathological results, while those in the adenoma polyp group had adenoma polyps, adenocarcinoma, papilloma, and xanthomatous polyps. The patients in the adenoma polyp group were at high risk for carcinoma or had established carcinomatous polyps. Gallbladder specimens were fixed by formalin solution before pathological examination.

\section{Statistical analysis}

Descriptive statistics were used to calculate baseline characteristics and other clinical factors. The Pearson's correlation coefficient was used to show the association between polyp sizes calculated using radiographic modalities and those calculated using a pathological method. If it was significant, a multivariate linear regression model was used to predict pathological polyp sizes according to various clinical factors including the imaging size of the polyp. This model was implemented in all three groups. Results are shown as coefficients, standard error, $P$-values, and $R$ square of the final model. All statistical analyses were performed using STATA software, version 10.1 (College Station, TX, USA).

\section{Ethical considerations}

The study protocol was approved by the ethics committee in human research, Khon Kean University. Patient consent to review their medical records was not required by the institutional review board because the study was a retrospective study and the study does not involve identifiable individuals. Patient data confidentiality was protected and accorded with the principles of the Declaration of Helsinki.

\section{Results}

During the study period, 85 patients met the study criteria. The patients had a mean age of 51.6 years and $44.7 \%$ were male (Table 1). The average body mass index was $25.1 \mathrm{~kg} / \mathrm{m}^{2}$ and dyslipidemia was the most common co-morbidity $(8.5 \%)$. Abdominal pain was the most common presenting symptom (36.5\%), while $31.8 \%$ of patients were asymptomatic. Laboratory results were mostly within the normal range. Ultrasonography was performed in most of the patients (70 patients; $82.4 \%$ ), while the other patients underwent computed tomography (six patients; 7.1\%), ultrasonography plus computed tomography (five patients; 5.9\%), and ultrasonography plus magnetic resonance cholangiopancreatography (four patients; 4.7\%).

The average size of polyps according to radiographic modalities was slightly larger than the average pathological

Table I Baseline characteristics of patients who underwent laparoscopic cholecystectomy $(\mathrm{n}=85)$

\begin{tabular}{|c|c|}
\hline Factor & Value $(n=85)$ \\
\hline Age (years) & $51.6 \pm 12.1$ \\
\hline Male gender & $38(44.7)$ \\
\hline Married & $77(90.6)$ \\
\hline BMI $\left(\mathrm{kg} / \mathrm{m}^{2}\right)$ & $25.1 \pm 3.7$ \\
\hline \multicolumn{2}{|l|}{ Co-morbid disease } \\
\hline Diabetes & $2(2.8)$ \\
\hline Hypertension & $5(7.0)$ \\
\hline Dyslipidemia & $6(8.5)$ \\
\hline Number of children & $4.5(1.5)$ \\
\hline Family history of cancer & $2(2.4)$ \\
\hline \multicolumn{2}{|l|}{ Presenting symptom } \\
\hline Asymptomatic & $27(31.8)$ \\
\hline Pain & $31(36.5)$ \\
\hline Discomfort & $23(27.1)$ \\
\hline Burning & $2(2.4)$ \\
\hline Hematocrit (\%) & $38.6 \pm 4.1$ \\
\hline White blood cells $\left(/ \mathrm{mm}^{3}\right)$ & $7,382 \pm 1,584$ \\
\hline Platelets $\left(10_{9} / \mathrm{L}\right)$ & $259 \pm 81.8$ \\
\hline PMNs (\%) & $56.7 \pm 10.2$ \\
\hline Serum creatinine (mg/dL) & $0.9 \pm 0.9$ \\
\hline Serum albumin $(g / d L)$ & $4.3 \pm 0.4$ \\
\hline Serum ALT (U/L) & $28.4 \pm 19.0$ \\
\hline Serum AST (U/L) & $28.2 \pm 18.0$ \\
\hline Imaging size (mm) & $7.2 \pm 4.4$ \\
\hline Pathological size (mm) & $5.6 \pm 6.4$ \\
\hline \multicolumn{2}{|l|}{ Pathological type } \\
\hline Cholesterol polyp & $66(77.6)$ \\
\hline Adenoma polyp & $19(22.4)$ \\
\hline
\end{tabular}

Note: Data are shown as mean \pm SE or $n(\%)$.

Abbreviations: BMI, body mass index; PMN, polymorphonuclear leukocyte; ALT, alanine aminotransferase; AST, aspartate aminotransferase. 
size (7.2 vs $5.6 \mathrm{~mm})$. The majority were cholesterol polyps (66; 77.6\%). By radiography, the average (range) sizes of cholesterol and adenoma polyps were $6.6(3-20) \mathrm{mm}$ and 9.2 (4-20) $\mathrm{mm}$, respectively, the average (range) sizes of cholesterol and adenoma polyps by pathology were 4.2 (1-20) $\mathrm{mm}$ and 8.7 (1-35) mm, respectively. The Pearson's correlation coefficients showed significant correlation between the imaging sizes and pathological sizes of the polyps in all three groups $(P<0.01)$. The Pearson's correlation coefficient was highest in the adenoma group (0.9547), followed by the all-patient group (0.778), and was lowest in the cholesterol group (0.5744).

The size of the polyps according to radiographic imaging was significantly associated with pathological size in all three groups (Tables 2-4). The highest coefficient was in the adenoma group (1.706), as shown in Table 4. Age was another significant factor related to polyp size as identified

Table 2 Factors associated with pathological polyp size by multivariate linear regression analysis in all patients $(n=85)$

\begin{tabular}{llll}
\hline Factor & Coefficient & SE & $\boldsymbol{P}$ \\
\hline Age & 0.143 & 0.086 & 0.110 \\
Male gender & 0.843 & 1.933 & 0.666 \\
BMI & -0.266 & 0.279 & 0.349 \\
Imaging size & 1.110 & 0.218 & $<0.001$ \\
PMNs & -0.028 & 0.110 & 0.803 \\
\hline
\end{tabular}

Note: $R^{2}=0.6481$.

Abbreviations: BMI, body mass index; PMN, polymorphonuclear leukocyte.

Table 3 Factors associated with pathological polyp size by multivariate linear regression analysis in patients with cholesterol polyps $(n=66)$

\begin{tabular}{llll}
\hline Factor & Coefficient & E & $\boldsymbol{P}$ \\
\hline Age & -0.062 & 0.085 & 0.478 \\
Male gender & 2.014 & 1.699 & 0.252 \\
BMI & -0.196 & 0.234 & 0.415 \\
Imaging size & 0.568 & 0.222 & 0.021 \\
PMNs & -0.012 & 0.098 & 0.903 \\
\hline
\end{tabular}

Note: $R^{2}=0.4392$.

Abbreviations: BMI, body mass index; PMN, polymorphonuclear leukocyte.

Table 4 Factors associated with pathological polyp size by multivariate linear regression analysis in patients with adenoma polyps $(n=19)$

\begin{tabular}{llll}
\hline Factor & Coefficient & SE & $\boldsymbol{P}$ \\
\hline Age & 0.264 & 0.039 & 0.021 \\
Male gender & 3.440 & 0.998 & 0.075 \\
BMI & -0.235 & 0.161 & 0.283 \\
Imaging size & 1.706 & 0.128 & 0.006 \\
PMNs & 0.192 & 0.052 & 0.065 \\
\hline
\end{tabular}

Note: $R^{2}=0.9974$.

Abbreviations: BMI, body mass index; PMN, polymorphonuclear leukocyte. using the pathological method in the adenoma group, with a coefficient of $0.264(P=0.021)$.

\section{Discussion}

This study confirmed that polyp size according to radiographic modalities (mostly ultrasonography) was a significant predictor for pathological size in all patients and in those with cholesterol polyps and those with adenoma polyps. However, polyps measured using radiography appeared to be smaller than those measured using pathological methods after adjustment for other clinical factors. The average size of the adenoma polyps by pathological evaluation was 1.706 $\mathrm{mm}$ larger than that of those measured using radiographic methods (Table 4). However, the average size of cholesterol polyps using pathological methods was only $0.568 \mathrm{~mm}$ larger than that of those measured using radiographic methods (Table 3). It has previously been reported that ultrasonography is an inaccurate method to differentiate among causes of gallbladder polyps. ${ }^{10}$ The sensitivity of ultrasound to detect carcinomatous polyps was $52.4 \%$. These findings may explain why measurements were an average of $1.706 \mathrm{~mm}$ larger in the adenoma group per $1 \mathrm{~mm}$ of gallbladder polyp seen by ultrasound. Formalin fixation may be another factor associated with shrinkage of polyp size. A previous report found that formalin fixation reduced pathological sizes by $4.10-6.18 \% .{ }^{11}$ In this study, the pathological size was reduced from radiographic findings by $22.2 \%$ (from 7.2 to $5.6 \mathrm{~mm}$ ). Therefore, it is unlikely that the smaller pathological size can be explained by only formalin fixation.

Advanced age has been reported to be associated with malignant gallbladder polyps. ${ }^{2,4}$ Even though the incidence of gallbladder polyp cancer was low, at $0.57 \%,{ }^{12}$ increasing age is one important risk factor for cancer development. A study from China found that age over 50 years increased the risk of malignant polyps by 16.5 times. $^{2}$ Similarly, this study also found that for every 1 year increase in age, the average size of adenoma gallbladder polyps (but not cholesterol polyps) increased by $0.264 \mathrm{~mm}$ (Tables 3 and 4 ). Note that the presence of adenoma polyps in this study was not considered to indicate cancer, but may represent a pre-cancerous condition.

There are some limitations in this study. First, it used a very specific study population. All patients underwent laparoscopic cholecystectomy, not open cholecystectomy. However, the results may not differ greatly between the two surgical procedures. Second, most gallbladder polyps in this study were cholesterol polyps (77.6\%). Finally, the sample size was small. Despite this, the results reached statistical significance. To our knowledge, there have been few studies 
in recent years that have attempted to predict pathological polyp size using clinical variables in addition to radiographic imaging (mostly ultrasonography). Further larger studies are needed.

In conclusion, gallbladder polyp size as measured by radiographic modalities can predict pathological size in both cholesterol and adenoma polyps. Some adjustments are needed, particularly in the case of adenoma polyps.

\section{Acknowledgments}

The authors would like to thank Sleep Apnea Research Group, Research Center in Back, Neck and Other Joint Pain and Human Performance, and Research and Training Center for Enhancing Quality of Life of Working Age People, Khon Kaen University, for their kind support.

\section{Disclosure}

The authors report no conflicts of interest in this work.

\section{References}

1. Sarici IS, Duzgun O. Gallbladder polypoid lesions $>15 \mathrm{~mm}$ as indicators of T1b gallbladder cancer risk. Arab J Gastroenterol. 2017;18(3):156-158.

2. Xu A, Zhang Y, Hu H, Zhao G, Cai J, Huang A. Gallbladder Polypoid-Lesions: What Are They and How Should They be Treated? A Single-Center Experience Based on 1446 Cholecystectomy Patients. J Gastrointest Surg. 2017;21(11):1804-1812.
3. Wiles R, Thoeni RF, Barbu ST, et al. Management and follow-up of gallbladder polyps : Joint guidelines between the European Society of Gastrointestinal and Abdominal Radiology (ESGAR), European Association for Endoscopic Surgery and other Interventional Techniques (EAES), International Society of Digestive Surgery - European Federation (EFISDS) and European Society of Gastrointestinal Endoscopy (ESGE). Eur Radiol. 2017;27(9):3856-3866.

4. Guo J, Wu G, Zhou Z. Polypoid lesions of the gallbladder: report of 160 cases with special reference to diagnosis and treatment in China. Int J Clin Exp Pathol. 2015;8(9):11569-11578.

5. Babu BI, Dennison AR, Garcea G. Management and diagnosis of gallbladder polyps: a systematic review. Langenbecks Arch Surg. 2015;400(4):455-462.

6. Choi YS, do JH, Seo SW, et al. Prevalence and Risk Factors of Gallbladder Polypoid Lesions in a Healthy Population. Yonsei Med J. 2016;57(6):1370-1375.

7. Velidedeoğlu M, Çitgez B, Arıkan AE, Ayan F. Is it necessary to perform prophylactic cholecystectomy for all symptomatic gallbladder polyps diagnosed with ultrasound? Turk J Surg. 2017;33(1):25-28.

8. Ahmed M, Diggory R. The correlation between ultrasonography and histopathology in the management of gall bladder polyps. Acta Chir Belg. 2013;113(3):208-212.

9. Kim JH, Lee JY, Baek JH, et al. High-resolution sonography for distinguishing neoplastic gallbladder polyps and staging gallbladder cancer. AJR Am J Roentgenol. 2015;204(2):W150-W159.

10. Azuma T, Yoshikawa T, Araida T, Takasaki K. Differential diagnosis of polypoid lesions of the gallbladder by endoscopic ultrasonography. $\mathrm{Am}$ J Surg. 2001;181(1):65-70.

11. Chen CH, Hsu MY, Jiang RS, Wu SH, Chen FJ, Liu SA. Shrinkage of head and neck cancer specimens after formalin fixation. $J$ Chin Med Assoc. 2012;75(3):109-113.

12. Elmasry M, Lindop D, Dunne DF, Malik H, Poston GJ, Fenwick SW. The risk of malignancy in ultrasound detected gallbladder polyps: A systematic review. Int J Surg. 2016;33(Pt A):28-35.
Reports in Medical Imaging

\section{Publish your work in this journal}

Reports in Medical Imaging is an international, peer-reviewed, open access journal publishing original research, reports, reviews and commentaries on all areas of medical imaging. The manuscript management system is completely online and includes a very quick and fair peer-review system, which is all easy to use.

\section{Dovepress}

Visit http://www.dovepress.com/testimonials.php to read real quotes from published authors. 